\title{
Erratum to: Finite Volumes for Complex Applications VIII-Hyperbolic, Elliptic and Parabolic Problems
}

\author{
Clément Cancès and Pascal Omnes
}

\section{Erratum to:}

C. Cancès and P. Omnes (Eds.), Finite Volumes

for Complex Applications VIII-Hyperbolic, Elliptic and Parabolic Problems, Springer Proceedings in Mathematics \& Statistics 200, https://doi.org/10.1007/978-3-319-57394-6

The original version of the book was inadvertently published with incorrect table, figure position and equation, which have been corrected as follows

In Chap. 9, Table 1, incomplete table lines have been corrected and the text "N/A" has been center aligned by merging all the empty cells.

In Chap. 37, the position of sub-figures of Fig. 4 has been interchanged.

In Chap. 54, the equation $b_{\vec{u}, T}\left(\underline{u}_{T}, \underline{v}_{T}\right):=\left(u_{T}, \mathrm{G}_{\vec{u}, T}^{k+1} \underline{u}_{T}, v_{T}\right)_{T}+s_{\vec{u}, T}\left(\underline{u}_{T}, \underline{v}_{T}\right)$ has been corrected as $-b_{\vec{u}, T}\left(\underline{u}_{T}, \underline{v}_{T}\right):=-\left(u_{T}, \mathrm{G}_{\vec{u}, T}^{k+1} \underline{v}_{T}\right)_{T}+s_{\vec{u}, T}\left(\underline{u}_{T}, \underline{v}_{T}\right)$ 